\title{
Peripersonal space boundaries around the lower limbs
}

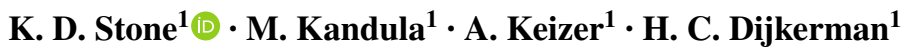

Received: 9 June 2017 / Accepted: 27 October 2017 / Published online: 2 November 2017

(C) The Author(s) 2017. This article is an open access publication

\begin{abstract}
Neurophysiological investigations in non-human primates have shown that bi- and tri-modal fronto-parietal neurons exist that respond to touch on the body and visual (and/or auditory) stimuli near the body. The receptive fields of these neurons extend into space around the body, producing a zone wherein multisensory information is readily integrated. This space around the body, known as peripersonal space (PPS), has also been investigated behaviourally in humans. Some studies have focused on how far into depth the spatial boundaries of PPS extend. Most of these investigations have focused on the upper body (e.g., hands, face, trunk), while little is known about the size of PPS for the lower body (i.e. legs and feet). Thus, the aim of the current study was to delineate a PPS boundary around the lower limbs in healthy participants using a multisensory interaction task. Participants made speeded responses to the presence of vibrations applied to the toes while a taskirrelevant visual stimulus approached towards (Experiment 1) or receded from (Experiment 2) the feet. Participants responded significantly faster to tactile stimuli when the visual stimulus was within approximately $73 \mathrm{~cm}$ from the feet, but only when it approached (and not receded from) the legs. This is the first study, to our knowledge, to outline the size of PPS for the lower limbs. These findings could provide insight into the mechanisms underlying multisensory
\end{abstract}

Electronic supplementary material The online version of this article (https://doi.org/10.1007/s00221-017-5115-0) contains supplementary material, which is available to authorized users.

\footnotetext{
K. D. Stone

k.d.stone@uu.nl

1 Department of Experimental Psychology, Helmholtz Institute, Utrecht University, Heidelberglaan 1, 3584 CS Utrecht, The Netherlands
}

integration in the lower limbs, and add to the current body of knowledge on PPS representations.

Keywords Visuo-tactile integration - Peripersonal space . Perception $\cdot$ Lower body

\section{Introduction}

In daily life, we encounter many instances where we must interact with objects in the space near our bodies. For instance, one might swat an approaching (buzzing) fly away from his face, or retract the foot from an oncoming wave on the beach shore. Intriguingly, early neurophysiological studies in non-human primates have shown that there are special fronto-parietal neurons that respond not only to an object touching a body part but also the presence of an object near that same body part (Graziano et al. 1994, 1997; Fogassi et al. 1996; Graziano and Cooke 2006). These neurons are bi- and tri-modal in nature; their tactile receptive fields overlap with visual and/or auditory receptive fields that extend into space around the body. The product of these extended receptive fields is known as peripersonal space (PPS; Rizzolatti et al. 1981a), a predictive multisensory integration zone around the body (Kandula et al. 2015). Noteworthily, this system is particularly sensitive to dynamic, compared to static, stimuli (Fogassi et al. 1996). And specifically, it is more responsive to stimuli approaching the body than to stimuli receding away from the body (Colby et al. 1993; Graziano and Gross 1995; Fogassi et al. 1996; Graziano et al. 1997; Duhamel et al. 1998). This space ultimately serves as a defense network (Cooke and Graziano 2003, 2004; Graziano and Cooke 2006) and sensorimotor interaction area for the body (Rizzolatti et al. 1997; Makin et al. 2008; Brozzoli et al. 2010). Thus, in the PPS network, the same 
neuron that responds to the buzzing fly touching your face also responds to the buzzing fly approaching your face. How far the receptive fields of these neurons extend into depth provides insight into the size of the PPS representation for different body parts. The edge of this representation is where the boundary between PPS and extrapersonal space (EPS: outside of PPS) lies. In monkeys, PPS extends (usually) to approximately $30 \mathrm{~cm}$ for the upper (head, hands, arms, upper torso) body, but can range between 5 and $100 \mathrm{~cm}$ (Colby et al. 1993; Graziano et al. 1994, 1999; Graziano and Gross 1995). In other words, once an approaching object arrives within $30 \mathrm{~cm}$ of the body, the discharge rate of these bimodal neurons is enhanced (Graziano et al. 1997). In turn, the size of the PPS representation for a body part can be defined by the activity of these neurons. However, the PPS representation can also be defined using behavioural paradigms.

For example, behavioural and imaging studies have suggested a similar fronto-parietal PPS network in humans, consisting mostly of the superior parietal, temporo-parietal and ventral premotor areas (Bremmer et al. 2001; Makin et al. 2007; Serino et al. 2011; Gentile et al. 2011; Brozzoli et al. 2012a, 2013; Cléry et al. 2015; Grivaz et al. 2017). We can examine the PPS representation behaviourally in humans using multisensory interaction paradigms. Using these types of paradigms, it has been shown that tactile detection (in terms of sensitivity, accuracy, and speed) on a body part is enhanced when a visual (or auditory) stimulus is presented near, as opposed to far from, the body. Evidence for this arises from studies involving stroke patients with tactile extinction (i.e., the inability to detect tactile stimuli on the contralesional side of the body when another stimulus is presented simultaneously on the ipsilesional side). Ladavas and colleagues have shown that extinction of a tactile stimulus on the contralesional side can be diminished (i.e., participants are more accurate at detecting the tactile stimulus) when a visual stimulus is also presented near that same side, but only when it is presented near, and not far from, the body [Làdavas et al. 1998, but see Làdavas et al. (2001) for a case with auditory information]. Similarly, in the healthy participants, Salomon et al. (2017) recently showed that the presence of irrelevant (and even consciously invisible) visual stimuli within, but not outside of, one's PPS can enhance sensitivity to noisy, near-threshold, tactile stimulation on the body. Again, this highlights how tactile detection is enhanced within PPS.

One's boundary between peri- and extra-personal space can be approximated by asking participants to respond to tactile input while in the presence of irrelevant looming auditory or visual information (Canzoneri et al. 2012, 2013b; Noel et al. 2015; Serino et al. 2015; de Haan et al. 2016; Kandula et al. 2017), as the PPS network is particularly sensitive to dynamic (especially approaching) sensory information. In such a paradigm, a tactile stimulus (vibration) is applied to the participants' limb at different time points along the trajectory of a looming auditory or visual stimulus, and participants are asked to respond (as quickly as possible) to the tactile stimulus only. Many studies have shown that tactile detection (in terms of speed and sometimes sensitivity) is modulated by the proximity of the approaching (albeit irrelevant) visual (or auditory) stimulus to the body (even though the participant is asked to ignore the visual (auditory) information). In these tasks, the relationship between the location of the visual stimulus and the tactile reaction times is usually sigmoidal in nature. That is, there is a point in space (i.e., the central point of the sigmoid) wherein tactile reaction times are suddenly reduced during the approaching trajectory of a visual or auditory stimulus. This 'spatial limit' where tactile reaction times are sped up is defined as the PPS boundary (Canzoneri et al. 2012). Critically, these reaction times within the approximated PPS are significantly faster than reaction times to unimodal tactile stimulation (i.e., when no visual stimulus is presented in conjunction with the tactile stimulus). Notably, when the stimulus instead recedes from the body, this sigmoidal relationship (usually) disappears (Canzoneri et al. 2012; Teneggi et al. 2013; Noel et al. 2015; Serino et al. 2015).This aligns with the aforementioned neurophysiological studies that have shown that neurons in the PPS network preferentially respond to stimuli approaching (in contrast to receding from) the body (Colby et al. 1993; Graziano and Gross 1995; Fogassi et al. 1996; Graziano et al. 1997; Duhamel et al. 1998).

Most investigations concerning the size of the PPS representation in humans have focused on the upper body (hands, head, trunk). The boundary between PPS and EPS for these body parts has been approximated to be around $30-50 \mathrm{~cm}$ for the hands and $60 \mathrm{~cm}$ for the head using the paradigm described above [see Serino et al. (2015) for a comprehensive examination of body part-centered PPSs]. With respect to the trunk (i.e., upper back/chest), its PPS representation tends to extend much further into space when compared to the hands or head, ranging from 65 to $100 \mathrm{~cm}$. The representation extends even further (up to $166 \mathrm{~cm}$ from the body) when participants walk on a treadmill during the task [thus, also engaging their lower limbs (Noel et al. 2015)]. This not only suggests an intimate relationship between the representation of peri-trunk and peri-leg/foot space but also reminds us that movement (or use) of the legs is almost always associated with a visually 'looming' environment, which is particularly relevant for the PPS network. It is not surprising, however, that the trunk PPS is modulated also by movement of the legs, as both body parts are involved in full-body actions. Serino et al. (2015) speculate that body parts responsible for "whole-body actions" might have tactile-receptive fields with size-appropriate overlapping visual (or auditory)receptive fields that extend even further into space than 
body parts whose action space is more confined (e.g., hands are confined to arm space). In other words, it seems that the size of the PPS around a body part is (at least partly) related to the action produced by the specific body part, rendering PPS not only a defensive space but also an action space [see de Vignemont and Iannetti (2015) for a stimulating discussion on the matter]. But what remains unknown, however, is precisely how far the PPS extends into space for the lower limbs.

No study, to our knowledge, has investigated the boundary between PPS and EPS for the lower limbs (i.e., legs/ feet) specifically. However, recently, Stettler and Thomas (2016) showed that participants responded significantly faster to visual stimuli that were near (within stepping distance) as opposed to far (outside stepping distance) from the feet, alluding to the presence of a boundary between PPS and EPS for the feet. Yet, as only static visual stimuli were used, and tactile detection was not assessed, it was not specified at what point in space multisensory integration (i.e., the boundaries of PPS) might occur around the feet. Certainly, some investigations have focused on the integration of visual and tactile information within PPS around the feet (Schicke and Röder 2006; Schicke et al. 2009; Van Elk et al. 2013; Scandola et al. 2016). For example, Schicke et al. (2009) were one of the first groups to show (behaviourally) that a PPS representation around the feet exists using a crossmodal congruency task. In this paradigm, participants are asked to make judgements about the elevation of tactile input on two points of one body part, while ignoring stationary visual stimuli that simultaneously appear in the same or different location (albeit still near the body's surface) as the tactile input. Tactile processing, measured via reaction times, is boosted when the visual and tactile information appear at the same location, at the same time (suggesting that the brain, indeed, cannot simply ignore the visual information), known as the crossmodal congruency effect (CCE). When the visual stimulus is spatially incongruent with the tactile stimulus, then tactile reaction times are significantly slowed. The results of Schicke and colleagues revealed that the feet exhibit a CCE that is of similar magnitude to that of the hands, suggesting that PPS around the feet indeed exists. Further evidence for a lower limb PPS representation comes from the work of Noel and Wallace (2016). Participants were asked to judge the order of stimulation for vibrations applied to both ankles (while they were crossed or uncrossed) under normal and sensory-deprived (i.e., no vision, no auditory, or no vision and auditory) conditions. They showed that depriving participants of audio and visual information (albeit irrelevant to the task) impaired spatial localization of tactile information on the feet, particularly when the legs were crossed, suggesting that spatial localization of the lower body critically depends on multisensory integration. These findings provide compelling evidence that a peri-foot representation exists, and thus suggest that mapping its size is a feasible aim.

It is surprising that very few investigations have focused on the size of the PPS representation for the lower limbs. For instance, one of the main proposed functions of PPS is to 'provide a margin of safety' around the body (Graziano and Cooke 2006). If someone intrudes this margin of safety (i.e., comes too close to us), we will usually step away from the individual to re-establish this boundary (Graziano and Cooke 2006). The stepping action is directly guided by the lower limbs, and perhaps it is modulated by their (or the whole body's) PPS representation. But while Graziano et al. (1997) suggested that 'foot-centered' PPS neurons likely exist, PPS boundaries around the lower limbs were never (neurophysiologically) investigated. One possibility (as alluded to) is that the lower limb PPS representation aligns with the trunk's PPS representation, as both the trunk and the feet are intimately linked to full body actions (such as walking or balance). Still, the lower limbs, although anatomically connected to, are physically distinct from the trunk. Furthermore, they have a larger, more flexible action space when compared to the trunk, and this might be reflected in the size of their PPS representation. In any case, an investigation of the size of PPS around the lower limbs is lacking in the current literature.

Therefore, the goal of the current study was to investigate the size of the PPS representation for the lower limbs. Specifically, we aimed to delineate a boundary between peri- and extra-personal space for the lower limbs using a multisensory (visuo-tactile) interaction paradigm. To investigate the role of (stationary) tactile and (dynamic) visual information in outlining the PPS representation, we conducted two experiments. In Experiment 1, participants made judgements about the presence of a tactile stimulus while irrelevant visual stimuli approached the lower limbs (i.e., the feet). In Experiment 2, participants made judgements about the presence of a tactile stimulus while an irrelevant visual stimulus receded away from the lower limbs. We expected that the size of the PPS representation for the lower limbs would be larger than for the upper body (as reported in previous studies), as the lower limbs are directly involved in moving the whole body. Moreover, we expected to find a boundary (i.e., sigmoidal relationship between reaction times and the location of the visual stimulus) for approaching, but not receding, visual stimuli as neurophysiological and behavioural studies have shown that the PPS network is particularly sensitive to looming stimuli.

\section{Methods: Experiment 1 and Experiment 2}

Note that methods for Experiment 1 and Experiment 2 are presented together. 


\section{Materials and procedures}

\section{Participants}

Experiment 1 Eighteen participants (16 female) between the ages of 19 and $33(M=23.3$ years, $\mathrm{SD}=3.7)$ took part. The average height of participants was $170 \mathrm{~cm}(\mathrm{SD}=7.1)$ with an average foot length of $23.9 \mathrm{~cm}(\mathrm{SD}=1.4)$. All participants had normal or corrected-to-normal vision, and reported having normal tactile sensitivity. Tactile sensitivity was assessed by asking the participant if he/she felt the vibrations during the practice trials. One additional participant was tested, but was removed from the dataset as he had an injured foot, and failed to respond to the tactile stimulus in $>10 \%$ of the trials.

Experiment 2 Nineteen participants (16 female) between the ages of 19 and $34(M=25.0$ years, $\mathrm{SD}=4.3)$ took part. The average height of participants was $170.5 \mathrm{~cm}(\mathrm{SD}=5.8)$ with an average foot length of $24.5 \mathrm{~cm}$ (SD = 1.1). All participants had normal or corrected-to-normal vision, and reported having normal tactile sensitivity. One additional participant was tested, but $35 \%$ of his responses were beyond two standard deviations for each tactile time point, and thus the data were not included in the analyses.

\section{Visuotactile-interaction task}

Experiment 1 To measure PPS boundaries, we used a visuo-tactile interaction task using dynamic visual stimuli [similar to the audio-visual interaction tasks in Canzoneri et al. (2012) and Serino et al. (2015) and to investigations in our lab: Kandula et al. (2017) and de Haan et al. (2016)]. The task and stimuli were designed and controlled by a custom MATLAB script. Tactile stimuli consisted of a $100 \mathrm{~Hz}$ vibration (duration of $100 \mathrm{~ms}$ ) produced by an $8 \mathrm{~mm}$ vibrotactile motor (Precision Microdrives, model: 308-00). Visual stimuli consisted of a $5 \mathrm{~cm}$ (diameter) red opaque circle that was projected onto the floor in front of the participant. Participants were instructed to respond (as quickly as possible) to tactile stimuli on their toes while the visual stimulus approached the toes.

Prior to task initiation, participants removed their shoes and socks. They were then seated comfortably at a chair with their knees bent at a $90^{\circ}$ angle and with their feet resting on a piece of black cloth (to provide comfort, $\sim 1 \mathrm{~cm}$ off of the floor). In front of the feet laid a rectangular board covered with a large opaque black cloth. A large mirror (100 cm L $\times 150 \mathrm{~cm} \mathrm{W,} \mathrm{attached} \mathrm{to} 80 \mathrm{~cm} \mathrm{H} \mathrm{legs),} \mathrm{bent}$ towards the floor at a $40^{\circ}$ angle, stood perpendicular (on the left side of the participant) to the rectangular board. Across from the mirror (165 $\mathrm{cm}$ away) stood a projector (model: SANYO ProXtraX, positioned on a stand, $110 \mathrm{~cm}$
$\mathrm{H}$ from the floor) which was responsible for stimulus projection (projection site: $60 \mathrm{~cm} \mathrm{~W} \times 143.5 \mathrm{~cm} \mathrm{~L}$ ). The stimuli were projected directly ahead into the bent mirror, and the mirror reflected the stimuli onto the cloth-covered rectangular board on the floor, directly in front of the participant. Therefore, we could create the illusion that visual stimuli were approaching towards (Experiment 1) or receding away (Experiment 2) from the feet. To reduce the possibility that participants might react to the sound, instead of the feeling, of the vibrating motors, participants listened to white noise via headphones for the duration of both experiments.

After the participant was seated, the experimenter attached two vibro-tactile motors to the surface of the participants' right and left big toes, respectively, on the skin directly below the toenails. The big toes were positioned $30 \mathrm{~cm}$ apart, as specified by two indicator dots projected onto the floor on the edge of the board on the floor. The motors were attached to a motor response box, positioned on a platform on the right side of the participant $(60 \mathrm{~cm} \mathrm{H})$. Participants rested their right hands on the box during the experiment, and were instructed to press a button on the box (as quickly as possible) using the index finger whenever they felt a vibration on either toe. The total duration of each trial was $8000 \mathrm{~ms}$. Before each trial began, participants were instructed to fixate on a cross presented in the center of the projection site (67 $\mathrm{cm}$ from the toes), which was present for 700-1000 ms. The fixation cross subsequently disappeared, and the projection remained blank for $2000 \mathrm{~ms}$. Then, a red circle starting moving (at $33.5 \mathrm{~cm} / \mathrm{s}$ ) from the far edge of the display (i.e., $138.5 \mathrm{~cm}$ from the toe). Once the center of the circle reached within $5 \mathrm{~cm}$ of the tip of the toe (i.e., after $4000 \mathrm{~ms}$ of travel), it disappeared. This was followed again by $2000 \mathrm{~ms}$ of a blank projection. In $50 \%$ of the trials, there was the possibility that the participant could receive one vibration (per trial) at 1 of 7 different time points. The first possible vibration could occur after the first $1000 \mathrm{~ms}$ (before the visual stimulus appeared on the screen). This vibration was coded as a unimodal tactile stimulus (because the stimulus occurred in absence of the visual stimulus). Thereafter, once the visual stimulus started moving, a single vibration could occur during one of the following time points: 2666.67, 3333.33, 4000, 4666.67 and $5333.33 \mathrm{~ms}$ (or, in distance, corresponding to every $22.23 \mathrm{~cm}$ of approaching movement). Thereafter, the final possible vibration could occur $1000 \mathrm{~ms}$ (at time point $7000 \mathrm{~ms}$ ) after the disappearance of the visual stimulus. This vibration was also coded as a unimodal tactile stimulus. Thus, a tactile stimulus could be administered in the presence of the moving visual stimulus at 1 of 5 possible locations (timepoints) from the feet (i.e., D5: $111.15 \mathrm{~cm}, D 4: 88.92 \mathrm{~cm}, D 3: 66.69, D 2: 44.46 \mathrm{~cm}$, or $D 1: 22.23 \mathrm{~cm}$ ) or in the absence of the visual stimulus (unimodal trials) at 1 of 2 possible time points (i.e., at the beginning or end of the trial). The unimodal trials were used 
to calculate a baseline for tactile reaction times. This baseline was used to investigate if tactile reaction times were facilitated by the visual stimulus in the visual-tactile trials. In the remaining $50 \%$ of the trials, no tactile stimulus was given (catch trials). The reason for this manipulation was to control for the effects of expectancy during the task, which has been shown to modulate the activity of cortical sensorimotor areas [thereby affecting response times; e.g. Haegens et al. (2011)]. Moreover, recent investigations in our lab have revealed that the optimal ratio of real to catch trials in this paradigm is 1:1 (Kandula et al. 2017). The real and catch trials were intermixed throughout the experiment, and randomized between participants. Participants completed 168 (84 trials per foot) trials, separated into 3 separate blocks (56 trials/block). Additionally, participants completed ten practice trials (not included in the analysis) before the initiation of the experiment to become familiarized with the task. Therefore, participants received six tactile stimuli per time point, per foot. See Fig. 1 for visualization of the procedures. As we were interested in PPS around the lower limbs in general, the reaction times were collapsed across foot. Analyses comparing reaction times per foot are included in the supplementary material.

Experiment 2 Materials and procedures were the same as Experiment 1, except that the visual stimulus instead receded away from the toes rather than approached the toes.

\section{Data analysis}

\section{Experiment 1}

Data were analyzed using MATLAB (version R2015b) and SPSS (version 23). Normality was assessed by conducting Shapiro-Wilk tests, and by examining the skewness and kurtosis of each set. For normally distributed data, the mean is reported and variability is expressed as standard error of the mean (SE). For non-normally distributed data, the median is reported and variability is expressed as the interquartile range (IQR). Partial eta-squared values were used to show effect sizes $\left(\eta^{2}\right)$. When the assumption of sphericity was violated, the Greenhouse-Geisser correction was applied. Reaction times greater than two standard deviations from the individuals' mean reaction time for each tactile location were removed from the analysis. Moreover, trials wherein participants failed to respond to the tactile stimulus were not included in the analyses. Approximately, $6.8 \%$ of trials (wherein a response was required) were removed from the analyses. Moreover, a Pearson's correlation was run to assess the relationship between foot size and PPS size (i.e., the central point of each fitted sigmoid), given that PPS is body part-centered and could be related to limb size. Mean reaction times were fitted to two mathematical functions (sigmoidal and linear, equations described in next section) to examine which function described the data better using the Curve Fitting Toolbox in MATLAB. Furthermore,

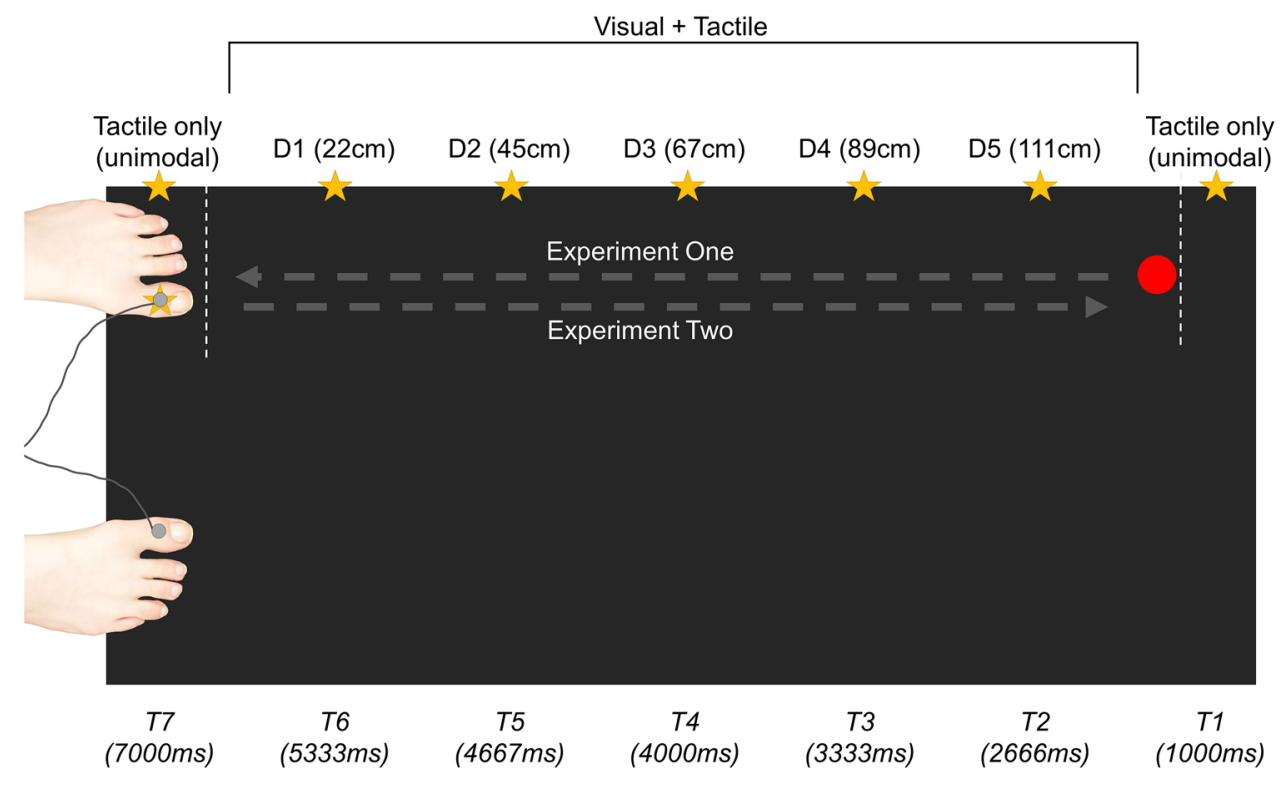

Fig. 1 Bird's eye view of stimulus set up. The large grey rectangle represents the stimulus projection site. The horizontal grey-dashed arrow indicates the visual stimulus (red circle) trajectory for Experiment 1 (approaching) and Experiment 2 (receding). The vertical white-dashed lines enclose the area in which the visual stimulus was on screen. The stars represent possible points in which a tactile stimulation to the toe could be given. $D$ represents visual stimulus distance from the toe and $T$ represents time in milliseconds from the start of the trial. For Experiment 2, the $T$ 's are reversed. Please note that $D$ 's and $T$ 's have been rounded to the nearest whole number 
repeated measures ANOVAs were conducted on the baseline-corrected reaction times for both experiments. To apply the baseline correction, we subtracted the tactile reaction time at each visual location from the individuals' fastest unimodal reaction time [as in Noel et al. (2015) and Serino et al. (2015)], to take the most conservative approach in comparing the reaction times to baseline. These comparisons to baseline allowed for an investigation of multisensory facilitation, a phenomenon where tactile reaction times are (significantly) sped up by the presence of visual information at different distances when compared to baseline. That is, the visual information facilitates the reaction times, depending on its proximity to the body.

\section{Experiment 2}

Analyses were the same as in Experiment 1. Similar to Experiment 1, approximately $6.2 \%$ of trials were removed from the analyses due to outliers and failed button presses.

\section{Results}

Note that results for Experiment 1 and Experiment 2 are presented together.

\section{Visuotactile-interaction task}

\section{Curve fitting}

To examine the relationship between tactile response times and visual stimulus location, we fitted the mean reaction times to both linear and sigmoidal functions [mimicking the analyses of Canzoneri et al. (2012), Ferri et al. (2015a, b), Serino et al. (2015), Kandula et al. (2017), and Taffou and Viaud-Delmon (2014)]. The fits of these functions were then compared to evaluate which function better described the data. If the data were better described by a linear function, with the $x$-axis representing the visual stimulus distance for the feet and the $y$-axis representing tactile reaction times, then this suggests that participants (consistently) responded faster to the tactile stimulus as the visual stimulus got closer (producing a diagonal line) or it suggests that participants consistently responded to the tactile stimulus at the same rate (regardless of the location of the visual stimulus), producing a horizontal line. In either case, no clear boundary between peri- and extra-personal space can be extracted. Alternatively, if the data is better described by a sigmoidal function, then this suggests that there was a point in space along the visual stimulus' approaching trajectory wherein reaction times to tactile stimulus are not consistently but instead suddenly enhanced, creating an s-shaped curve. This point in space (i.e., the central point of the sigmoid, or the "switch point') is the location where the boundary between periand extra-personal space presumably lies.

The equation for the linear function was as follows:

$y(x)=a \times x+b$,

where $y$ represents the reaction time to the tactile stimulus (in milliseconds), $a$ represents the slope, $x$ represents the location of the visual stimulus, and $b$ represents the $y$-intercept.

The equation for the sigmoidal function was as follows:

$y(x)=y_{\min }+\left(\frac{\left(y_{\max -} y_{\min }\right)}{\left(1+\mathrm{e}^{((a-x) \times b)}\right)}\right)$,

where $y$ represents the reaction time to the tactile stimulus (in milliseconds), $b$ represents the slope, $x$ represents the location of the visual stimulus, and $a$ represents the central point of the sigmoid.

In both models, we constrained the parameters so that there were only two that were free to vary: that is, slope and intercept. This allowed for direct (and fair) comparison between the two mathematical models. Goodness of fit was evaluated by comparing the root mean-squared errors (RMSEs) and $R^{2}$ values between both fitted functions. As these data were not normally distributed in both experiments, Wilcoxon signed-rank tests were conducted.

Experiment 1 (approaching stimuli) Wilcoxon signedrank tests revealed that the RMSEs for the sigmoidal fits $\left(\mathrm{Med}_{\text {sig }}=10.95 \pm 7.4-21.2 \mathrm{IQR}\right)$ were significantly lower than for the linear fits $\left(\operatorname{Med}_{\text {lin }}=11.98 \pm 9.7-\right.$ 25.5 IQR; $z=-2.1, p=0.03$ ), suggesting that the data were better described by the sigmoid function. Moreover, $R^{2}$ values were significantly higher for the sigmoidal $\left(\mathrm{Med}_{\text {sig }}=0.55 \pm 0.05-0.94 \mathrm{IQR}\right)$ when compared to the linear fits $\left(\mathrm{Med}_{\text {lin }}=0.45 \pm 0.05-0.77 \mathrm{IQR} ; z=-1.9, p=0.04\right)$, again, suggesting that the data were better explained by the sigmoid function. The mean of the central points of the individually fitted sigmoids was, therefore, used to determine the approximate boundary between PPS and EPS. The estimated boundary lies around $73.14 \mathrm{~cm} \pm 5.6 \mathrm{SE}$ from the feet (i.e., between distance 3 and distance 4). See Table 1 for values. See Fig. 2 for depiction of best fitting sigmoid at the group level.

Experiment 2 (receding stimuli) Wilcoxon signed-rank tests revealed no difference between the RMSEs for the sigmoidal $\left(\mathrm{Med}_{\text {sig }}=19.94 \pm 14.6-28.6 \mathrm{IQR}\right)$ and linear $\left(\mathrm{Med}_{\text {lin }}=22.22 \pm 14.8-27.9 \mathrm{IQR}\right)$ fits $(z=-0.6$, $p=0.52$ ). There was no difference between the $R^{2}$ values for the sigmoidal $\left(M_{\mathrm{sig}}=0.11 \pm 0.03-0.23 \mathrm{IQR}\right)$ and linear $\left(\mathrm{Med}_{\text {lin }}=0.12 \pm 0.03-0.25 \mathrm{IQR}\right)$ fits $(z=-0.6, p=0.54)$. See Table 1. 
Table 1 Medians of RMSE, $R^{2}$, and slope values for sigmoidal and linear fits in Experiment 1 and Experiment 2

\begin{tabular}{lcr}
\hline Experiment 1 & & \\
\hline Approaching & Sigmoid & Linear \\
\hline RMSE & $10.95^{*}$ & 11.98 \\
$R^{2}$ & $0.55^{*}$ & 0.45 \\
Slope & 1.19 & 5.65 \\
\hline Experiment & & Linear $(*)$ \\
\hline Receding & Sigmoid $\left(^{*}\right)$ & 22.22 \\
RMSE & 19.94 & 0.12 \\
$R^{2}$ & 0.11 & 1.77 \\
Slope & 0.18 & \\
\hline
\end{tabular}

Experiment 1: asterisks denote significant differences between sigmoidal and linear fits (i.e., for RMSE, $R^{2}$ values). Experiment 2: no significant differences were found between the two fits. Note the significant differences $\left(^{*}\right)$ between Experiment 1 and Experiment 2 on all parameters

${ }^{*} p<0.05$, denotes significant differences between sigmoid and linear fits (RMSE and $R^{2}$ values), within Experiments

$\left.{ }^{*}\right) p<0.05$, denotes significant differences between RMSE, $R^{2}$, and slope values between Experiment 1 and Experiment 2

Fig. 2 Best fitting sigmoid at the group level for Experiment 1 (approaching data). The plot represents group mean tactile reaction times for each visual stimulus location with respect to the toes (RMSE $=1.0$, $\left.R^{2}=0.99\right)$. Error bars represent standard error of the mean

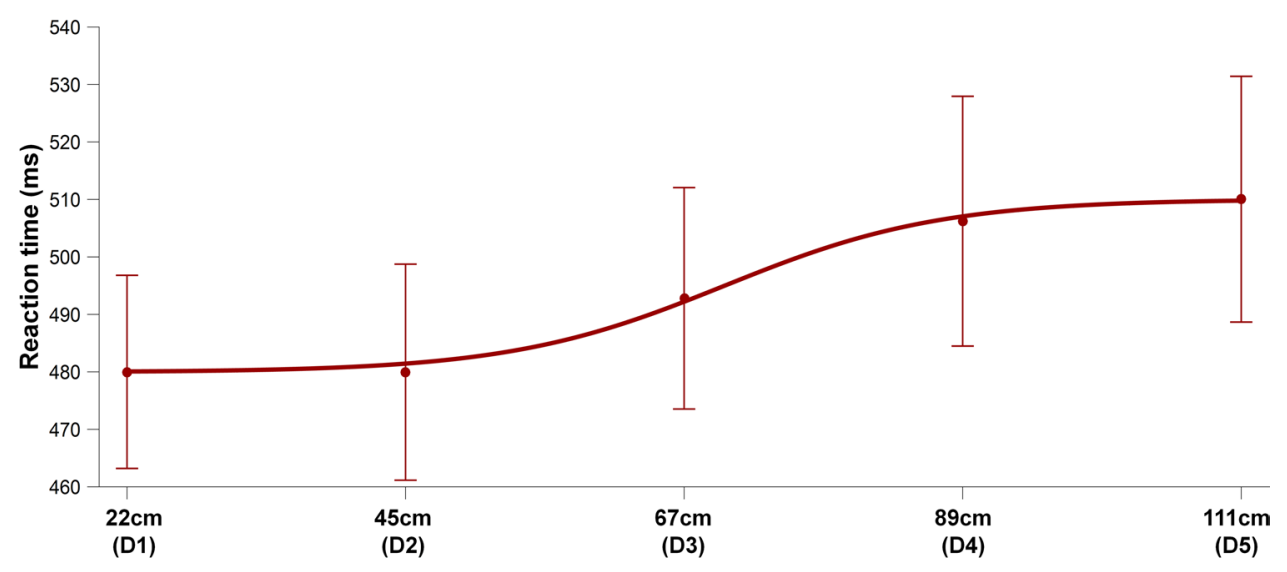

\section{Multisensory facilitation}

To complement the curve-fitting analyses, we also analyzed the baseline-corrected reaction times to allow for an investigation of multisensory facilitation between tactile reaction times and the different locations of the visual stimulus. Multisensory facilitation occurs when the tactile reaction times are significantly faster than baseline (e.g., Serino et al. 2015; Noel et al. 2015), suggesting that the tactile reaction times are facilitated by the visual information. This can be assessed for each distance (from near to far), and the distance wherein the corrected reaction times are no longer significantly different from baseline suggest that the PPS boundary lies somewhere between the previous (significantly different) distance and the distance that is not different from baseline. To reiterate, to apply the baseline correction, we subtracted the tactile reaction time at each visual location from the individuals' fastest unimodal reaction time [mimicking Noel et al. (2015) and Serino et al. (2015)].
Experiment 1 (approaching stimuli) Baseline-corrected reaction times were subject to a repeated measures ANOVA, with distance (D1-D5) as the within-subjects factor. There was, indeed, a main effect of distance $\left(F_{(4,68)}=5.0 ; p=0.007, \eta^{2}=0.228\right)$, indicating that tactile reaction times decreased as the visual stimulus got closer to the feet. To further investigate at which of these distances multisensory facilitation occurred, Bonferronicorrected one-sample $t$ tests (critical $p=0.01$ ) comparing the reaction times to baseline (i.e., $0 \mathrm{~ms}$ ) revealed that reaction times to the tactile stimulus were significantly faster than unimodal baseline at $D 1(22 \mathrm{~cm}, p=0.001)$, $D 2$ (44 cm, $p=0.001)$, and $D 3(67 \mathrm{~cm}, p=0.006)$, but not at $D 4$ or $D 5(p>0.09)$. Thus, this suggests that the boundary between peri- and extra-personal space for the lower body lies between D3 $(67 \mathrm{~cm})$ and $D 4(89 \mathrm{~cm})$. These results indeed complement the results of the curve fitting. See Fig. 3. 


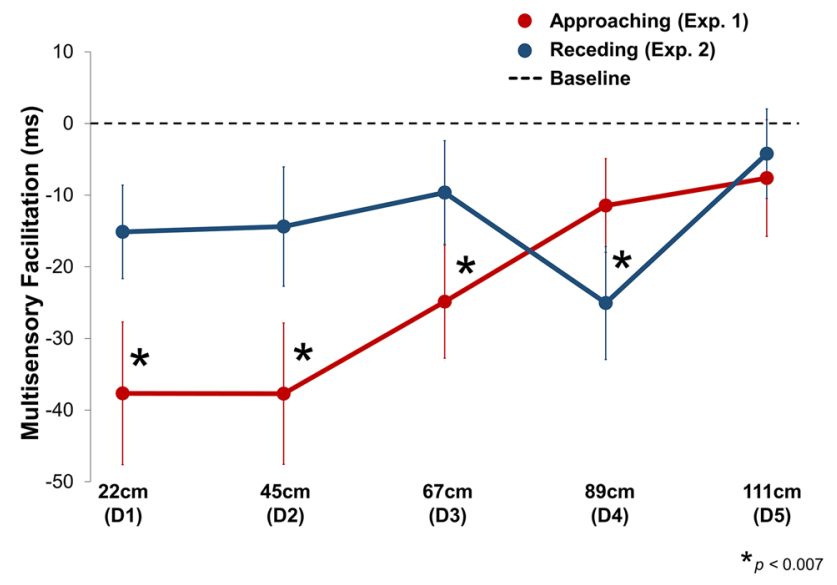

Fig. 3 Multisensory facilitation (in $\mathrm{ms}$ ) for the approaching group (Experiment 1, in red), and for the receding group (Experiment 2, in blue). Negative values indicate that reaction time for the visuo-tactile trials were faster than reaction times for the participants' fastest tactile only trials. Asterisks indicate significant differences from baseline (dashed line, $0 \mathrm{~ms}$ ). $D 1-D 5$ denote the proximity of the visual stimulus to the foot. For sake of clarity, distances are rounded to the nearest whole number. Note the significant difference for the approaching group at $D 1, D 2$, and $D 3$ (but not at $D 4$ or $D 5$ ) suggesting that the PPS boundary lay between $D 3$ and $D 4$. Moreover, this demonstrates a relationship between the proximity of the visual stimulus and the tactile reaction times. Note also the significant difference for the receding group at $D 4$. However, there is no clear relationship between the proximity of the visual stimulus and the tactile reaction times. Error bars represent standard error of the mean

Experiment 2 (receding stimuli) Baseline-corrected reaction times were subjected to a repeated measures ANOVA, with distance $(D 1-D 5)$ as the within-subjects factor. There was no main effect of distance $\left(F_{(4,72)}=2.3\right.$; $p=0.058, \eta^{2}=0.117$ ), however, the data revealed a reliable trend towards significance. To further investigate if there was multisensory facilitation at any of the distances, Bonferroni-corrected (critical $p=0.01$ ) one-sample $t$ tests comparing the reaction times to baseline (i.e. $0 \mathrm{~ms}$ ) suggested multisensory facilitation at $D 1(22 \mathrm{~cm}, p=0.03$, however, note that this did not survive the correction), but not at D2 $(p=0.1)$, nor at D3 or D5 $(p>0.19)$. Interestingly, however, there was facilitation at $D 4(89 \mathrm{~cm}$, $p=0.005)$. No clear estimated boundary, therefore, was found for receding stimuli. See Fig. 3 .

\section{Correlation between foot size and PPS size}

There was no correlation between foot size and PPS size for participants in Experiment $1[r(18)=0.38, p=0.11]$, nor between foot size and PPS size for participants in Experiment $2[r(19)=0.36, p=0.13]$.

\section{Visuotactile-interaction task: comparison between Experiment 1 and Experiment 2}

To investigate whether stimulus direction influenced tactile reaction times as a function of visual location, we ran two additional analyses comparing Experiment 1 and Experiment 2.

\section{Curve fitting}

We conducted separate Mann-Whitney $U$ tests comparing the RMSE, $R^{2}$, and slope values between the approaching (Experiment 1) and receding (Experiment 2) groups for both sigmoidal and linear fits. The analysis revealed that RMSEs for the sigmoidal fit were significantly lower for the approaching group compared to the receding group ( $U=94.0, p=0.01$ ). Moreover, $R^{2}$ values for the sigmoidal fit were significantly higher for the approaching compared to the receding group $(U=96.0, p=0.02)$. In addition, the slopes for the sigmoidal fit were significantly steeper for the approaching compared to the receding group $(U=93.0$, $p=0.01)$. In line with this, RMSEs for the linear fit were also significantly lower for the approaching group compared to the receding group ( $U=106.0, p=0.04$ ). Moreover, $R^{2}$ values for the linear fit were also significantly higher for the approaching compared to the receding group $(U=98.0$, $p=0.02$ ). In addition, the slopes of the linear fit were significantly steeper for the approaching group compared to the receding group $(U=99.0, p=0.03)$. See Table 1 .

\section{Multisensory facilitation}

Baseline-corrected reaction times were subject to a $5 \times 2$ repeated measures ANOVA with distance $(D 1-D 5)$ as the within-subjects factor and direction (approaching, receding) as the between-subjects factor. It should be noted that there were no significant differences in unimodal reaction times between Experiment 1 and Experiment 2 ( $p>0.1$ for both near and far administered tactile stimuli).

Results revealed a main effect of distance $\left(F_{(4,140)}=4.3\right.$; $\left.p=0.002, \eta^{2}=0.111\right)$, indicating that participants responded faster to the tactile stimulus when the visual stimulus was closer (rather than farther) from the feet. There was no main effect of direction $\left(F_{(1,35)}=1.3 ; p=0.24\right.$, $\left.\eta^{2}=0.038\right)$, indicating that overall, reaction times were not significantly different between the two experiments. However, the interaction between distance and direction was significant $\left(F_{(4,140)}=3.7 ; p=0.006, \eta^{2}=0.097\right)$. Follow-up Bonferroni-corrected paired samples $t$ tests (critical $p=0.0125$ ) revealed that the nature of this interaction likely arose from the differences observed between $D 4$ and $D 5$ for the receding group $[t(18)=-3.1, p=0.005$; mean difference $=-20.8 \mathrm{~ms}$, but not for the approaching group 
$[t(17)=-0.4, p=0.66$; mean difference $=-3.8 \mathrm{~ms}]$. This curious decrease in reaction times at $D 4$ in the receding group is visualized in Fig. 3.

\section{Discussion}

In the current study, we sought to uncover (and outline the size of) the peripersonal space representation for the lower body (legs and feet). Participants were asked to respond to a vibro-tactile stimulus on the toes which was administered at different time points during the trajectory of an approaching (Experiment 1) or receding (Experiment 2) visual stimulus. We found that reaction times to the tactile stimulus, for approaching visual stimuli only, showed a systematic decrease that was related to visual stimulus location. That is, the presence of the approaching visual stimulus boosted tactile processing within $73 \mathrm{~cm}$ (but multisensory facilitation remained present in the range of $67-89 \mathrm{~cm}$ ) from the toes. Thus, we were able to uncover a boundary between PPS and extrapersonal space (EPS). These findings extend our knowledge about PPS representations, and provide insight into multisensory integration around the feet.

Many studies that have examined PPS in humans have focused on the upper body (Canzoneri et al. 2012; Teneggi et al. 2013; Ferri et al. 2015a, b; Serino et al. 2015; Kandula et al. 2015, 2017; de Haan et al. 2016). This is logical as the early neurophysiological investigations in monkeys mainly focused on the PPS network governing the upper body (e.g., Cooke and Graziano 2003, 2004; Fogassi et al. 1996; Graziano 1999; Graziano et al. 1997, 1999; Rizzolatti et al. 1981b). However, neurons with receptive fields that extend into space for the whole body have also been identified (Hyvärinen 1981; Duhamel et al. 1998; Jiang et al. 2013). Further, some neurophysiological evidence in monkeys suggests that the dorsal areas of the ventral premotor cortex (Muakkassa and Strick 1979; Kurata et al. 1985; Kurata 1989) and medial regions of area 7 (Hyvärinen 1981) might be responsible for a 'foot-centered' PPS network, though an investigation using dynamic sensory stimuli was never formally reported. Thus, behavioural studies have uncovered the presence of PPS around the legs and feet (Schicke and Röder 2006; Schicke et al. 2009; Van Elk et al. 2013; Pozeg et al. 2015; Scandola et al. 2016; Stettler and Thomas 2016), but no study, to the best of our knowledge, has specifically examined how far PPS extends for the lower body.

There is indeed one recent study that looked at the influence of visual stimulus location on the spatial extent of foot PPS, though a metrically defined boundary between PPS and EPS was not delineated (Stettler and Thomas 2016). In this study, participants were asked to make speeded responses to the presence of a stationary visual stimulus (i.e., a square) which was positioned near (directly adjacent to the inner edge of the foot) or far (displaced approximately $20 \mathrm{~cm}$ laterally from the inner edge of the foot) from the foot. Participants responded significantly faster when the visual stimulus was near the foot, compared to when it was located at the 'far' adjacent side of the computer screen. Stettler and Thomas's study provides evidence for a lower limb PPS that is sensitive to visual stimulus location, a finding that was further reinforced in our current study. However, and in critical comparison to the current study, the direction (lateral to the feet vs. anterior to the feet), motion-related properties (stationary vs. dynamic) and number of investigated locations (2 vs. 6 ) of the visual stimulus differed. It is worth noting that PPS in the lateral direction is much less studied, and might be a fruitful avenue for upcoming research. Stettler and Thomas conclude that stimuli within 'stepping/kicking distance', although not explicitly measured or metrically defined in their report, are processed differently than those beyond this space. While we probably more often step forward, rather than in the lateral direction, the idea that stepping distance is relevant in delineating the size of PPS is an interesting proposal to also entertain in our current study. While we did not measure stepping distance in our sample, observations in adults of average weight and height have revealed that a typical step length is around $75 \mathrm{~cm}$ (Sekiya et al. 1997; Terrier and Schutz 2003; Terrier et al. 2005; Shorter et al. 2017). Remarkably, this distance aligns with the size of the PPS representation revealed in our study. While this idea requires a more thorough investigation, it lends strength to the proposal that PPS is, at least partly, a "multisensory motor-interface for body-object interaction" and is dependent upon a body part's 'action space' (Serino et al. 2009, 2015; Brozzoli et al. 2012b). There is at least one investigation that has focused on how the legs' 'potential for action' modulates their PPS representation. In this study, participants with spinal cord injury (SCI), who could not feel or move their legs, completed a crossmodal congruency task wherein vibrations were administered to the hands, and light flashes appeared near the feet (Scandola et al. 2016). To obtain a crossmodal congruency effect (CCE) in this case, the expectation is that tactile reaction times are facilitated when the visual stimulus is ipsilateral (congruent) to the tactile location, and conversely, hindered when the visual stimulus occurs contralateral (incongruent) to the tactile stimulus. Results revealed that individuals with SCI failed to show a CCE for the feet, but showed a normal CCE (and therefore PPS representation) for the hands. This finding suggests a retracted and/or absent PPS representation for their lower limbs. What is more, passively moving the participants' legs back and forth for only 15 min was enough to "reactivate" the PPS representation-as measured by the CCE—around their lower limbs. This remarkable finding 
highlights the role of the motor-related aspects in shaping PPS and also emphasizes the importance of studying the intricate mechanisms involved in how the brain represents the lower body. Although we cannot conclude that our current study supports, nor opposes, the proposal that lower limb PPS functions as a sensorimotor interaction zone, we hope that it, at least, adds further merit to this growing literature on lower limb representations and their PPS networks.

How does the size of the lower limb PPS representation revealed in the current study compare to the size of other body part-centered PPS representations? We found that the approximate boundary between PPS and EPS was located around $73 \mathrm{~cm}$ from the feet. As mentioned, most previous reports on PPS using behavioural paradigms have focused on the size of the peri-hand representation. This usually ranges between 30 and $50 \mathrm{~cm}$ from the hand (Canzoneri et al. 2013a; Serino et al. 2015; Kandula et al. 2017), but can be extended using a tool (Cardinali et al. 2009; Bassolino et al. 2010; Canzoneri et al. 2013b) or retracted after immobilizing the body part (Bassolino et al. 2014) or following an amputation (Canzoneri et al. 2013a). Therefore, how does the hand PPS representation compare to the lower body PPS representation? In one study, van Elk et al. (2013) showed that the foot PPS (as measured by a visuotactile crossmodal congruency task) is mostly unaffected by factors such as limb posture and the congruency of a fake limb placed nearby, whereas the hand PPS is sensitive to these factors. In addition, other studies suggest that the lower limbs are slower (when compared to the hands) at remapping tactile information (e.g., Schicke and Röder 2006). So it might not be surprising that the lower limb PPS size differs from the upper limb PPS size. One possibility is that these subtle differences between upper and lower body multisensory integration are related to the type of sensory-guided actions carried out by these limbs. As PPS is an action space, it is plausible that the body part's main action repertoire influences its PPS representation. For example, as acknowledged by Van Elk et al. (2013), visual information is, on a daily basis, more often coupled with proprioceptive information for the hands than for the legs (e.g., reaching to grasp a cup vs. walking). Reaching to grasp a cup requires vision (towards the hand and cup), touch, and proprioception, whereas walking does not require vision of the legs, but rather relies mainly on lower limb touch and proprioceptive feedback. In addition, many directed movements of the hands require precise attention and fine motor skills, whereas locomotion is guided by more gross, automatic movements, sometimes done in tandem with other body parts making goal-directed movements. For example, using the same paradigm as in the current study, Kandula et al. (2017) displayed a PPS boundary around the hands that was approximately $41( \pm \sim 7 \mathrm{SD}) \mathrm{cm}$. As we observe a larger (but more variable) PPS representation for the lower body 73 ( $\pm \sim 24 \mathrm{SD}$ ) $\mathrm{cm}$, we might speculate that these differences are partly reflective of the properties of the actions (e.g., precise vs. gross) conducted in these spaces.

In addition to peri-hand space, a few behavioural investigations have also focused on the size of peri-trunk space. The size of the peri-leg/foot space representation reported in the current study appears to be similar to that of the peritrunk space $(\sim 2 \mathrm{~cm}$, but ranging $65-100 \mathrm{~cm})$, as reported in previous studies (Noel et al. 2015; Serino et al. 2015). This is perhaps not surprising as the legs are physically connected and in direct line with the trunk. Movement of the legs, for example, during locomotion, simultaneously produces movement of the trunk whereas movement of the hands can occur independently from the rest of the body. Moreover, both the trunk and the legs are implicated in full-body object interactions (Noel et al. 2015; Serino et al. 2015). At first glance of our findings, it might appear that the trunk and lower limbs have a shared PPS. Serino et al. (2015) showed that body part-centered PPS depends on the body part's distance from the trunk. Utilizing a similar paradigm employed in our study, when the hand was positioned in the front and center of the trunk, the hand PPS was encapsulated by the trunk PPS. However, as the hand moved laterally away from the body, the hand PPS became smaller (and independent of the trunk's PPS), while the trunk PPS remained unchanged. It is possible that we captured both the trunk PPS and the lower limb PPS in the current study. It is noteworthy that in the current study, the lower limbs were not displaced laterally from the trunk but were indeed displaced in the anterior direction. The paradigm was designed so that the first point of possible collision of the visual stimulus with the body (and the site of vibro-tactile stimulation) was the toes. Because participants were sitting at a chair, with the feet slightly extended forward, the approximate anterior distance between the average participants' trunk and the tip of his/her toes was $75 \mathrm{~cm}$. Therefore, it is possible that the lower limb and trunk PPS (which has been measured while participants are standing) have similar metric distances, but separate PPS representations. What is more, locomotive movement of the legs has been shown to increase the size of the PPS (up to $1.66 \mathrm{~m}$ ) for the trunk (Noel et al. 2015). And although the authors mainly discuss these findings in terms of PPS plasticity, one might also entertain the possibility that the lower limb PPS network was also engaged during their task, and contributed to the noted PPS expansion. The activation of a PPS for paralyzed limbs following passive movement of only the legs in the report by Scandola and colleagues also suggests a lower limb PPS that is distinct from that of the trunk. However, as lower limb PPS has been seldom invested, these claims are merely speculative. In any case, we have shown that there is special space around the toes wherein visuotactile processing is facilitated. Future investigations could 
focus on further disentangling these body part-centered PPS representations (e.g., hand vs. foot, or trunk vs. foot).

We showed that visual stimulus direction seemed to play a critical role in defining the PPS boundaries for the lower limbs. In Experiment 1 (approaching visual stimuli), we found that the relationship between tactile reaction times and visual stimulus distance was better explained by a sigmoidal (s-shaped) function when compared to a linear function. This relationship was further confirmed by looking at the critical spatial distances from the feet where multisensory facilitation occurred, which allowed us to identify an approximate boundary between PPS and EPS. In general, the pattern of our data aligns with several previous reports that have identified a boundary between PPS and EPS for the hands (Canzoneri et al. 2012; Ferri et al. 2015a, b; Serino et al. 2015; Kandula et al. 2017), face (Sambo and Iannetti 2013; Teneggi et al. 2013; Serino et al. 2015), or the trunk (Galli et al. 2015; Noel et al. 2015; Serino et al. 2015). In contrast to Experiment 1, in Experiment 2 (receding visual stimuli), we found that neither the sigmoidal nor the linear function better described the relationship between tactile reaction times and visual stimulus distance. We were unable to identify a clear division between PPS and EPS when the visual stimulus receded away from the body. These findings not only match those of previous behavioural investigations on PPS size, but they also correspond with the neurophysiological studies that show that PPS neurons prefer to respond to approaching, over receding, stimuli (Colby et al. 1993; Graziano and Gross 1995; Fogassi et al. 1996; Graziano et al. 1997; Duhamel et al. 1998). And while some behavioural studies show that the peri-hand space is sensitive to stimuli approaching and receding from the hand (Canzoneri et al. 2012), PPS representations for other parts of the body, such as the face (Teneggi et al. 2013; Serino et al. 2015) or trunk (Serino et al. 2015) are diminished (or sometimes abolished) when stimuli recede away from the body part. However, and to our surprise, we did observe multisensory facilitation (i.e., significantly faster tactile reaction times compared to baseline) when the receding visual stimulus was located around $89 \mathrm{~cm}$ from the toes. Figure 3 (blue line) provides a visualization of this u-shaped dip, wherein reaction times significantly decreased from 67 to $89 \mathrm{~cm}$ from the body, and increased back to baseline from 89 to $111 \mathrm{~cm}$. This curious decrease in reaction times from 67 to $89 \mathrm{~cm}$ is strikingly reminiscent of the boundary between PPS and EPS that we found in Experiment 1. One might argue that, even when a stimulus is moving away from the body, it is still relevant to monitor its location with respect to the body [see Serino et al. (2015) for a brief point on this matter regarding interactions with the upper body]. After all, such as the arms, the legs are also important effectors for action. Not only do we interact with objects with the intent to move them away from our legs (e.g., kicking a ball) but, and maybe more rarely, we also make retracting movements with our legs (think of pulling the ball towards you in a game of soccer, or withdrawing the foot from an approaching spider). Perhaps the influence of receding stimuli on PPS representations is partly dependent on the level of bi-directionality of that body part (i.e., as we observed a limited effect of receding stimuli on the perileg space, which also has limited bi-directionality). Thus, it is plausible that the PPS network can be reactive to sensory information near, or on the cusp of, the boundary between PPS and EPS, regardless of stimulus direction. Of course, the brain must be able to distinguish between PPS and EPS to even formulate a boundary. Neuroimaging data shows that individual PPS boundaries can be predicted from intertrial variability in the BOLD signal for the premotor cortex when auditory stimuli are looming towards the hand, but only when the sounds are still located EPS (Ferri et al. 2015a). In other words, the PPS network is attentive to stimuli far from the body, before it even reaches PPS. So it is possible that reaction times to tactile stimuli are particularly sensitive around the PPS/EPS border, as the brain must maintain a representation of both spatial zones. Such a speculation could be challenged in future imaging studies, with the use of looming and receding stimuli.

Finally, it is worth acknowledging that we found a large spatial range that defined the boundary between PPS and EPS for approaching stimuli. The average slope from PPS to EPS was not sharp per se, but gradual. In other words, the switch from slower to faster reaction times, as the visual stimulus got closer, was elongated. This reflects the variability in PPS boundaries (or switch points) within the current sample. Studies have shown that individual differences in brain activity (Ferri et al. 2015a) and emotional states such as anxiety (Sambo and Iannetti 2013) or fear (Taffou and Viaud-Delmon 2014; Ferri et al. 2015b; de Haan et al. 2016), and also anxiety disorders such as claustrophobia (Hunley et al. 2017) can contribute to the variability observed in PPS boundaries. Although we did not specifically investigate this, it is possible that individual traits contributed to the large variability in our sample. But it is equally likely that the fluidity of the lower limb PPS boundary is also reflective of the frequency in which visual and tactile information are coupled for the lower body (Van Elk et al. 2013). As noted earlier, rarely do we need to look at our feet while walking, whereas we quite often look at our hand while making a grasp. Noel et al. (2016) showed that the window of time between two consecutive sensory stimuli that people judge them as occurring simultaneous is larger within PPS than outside of PPS. It would be interesting to compare these simultaneity judgements across body parts, particularly for the legs, to see if visual and tactile information are indeed integrated at a different rate. If so, this might affect the extent and precision of the PPS boundary around the lower body. 
To conclude, we believe we have uncovered a boundary between peri- and extra-personal space for the lower limbs using dynamic sensory stimuli. We hope that these findings lend insight into lower body multisensory processes and stimulate a plethora of new investigations involving the legs and feet. For instance, it would be interesting to compare these processes in individuals who experience the lower body differently from the general population (e.g., lower limb amputees, individuals with spinal cord injury, individuals with body integrity identity disorder).

Acknowledgements This study was supported by a Netherlands Organization for Scientific Research (NWO) Talent grant (406-15-282) to HCD, AK, and KDS, an NWO VICI Grant (453-10-003) to HCD, and a Natural Sciences and Engineering Research Council of Canada (NSERC) post-graduate scholarship to KDS.

Open Access This article is distributed under the terms of the Creative Commons Attribution 4.0 International License (http://creativecommons.org/licenses/by/4.0/), which permits unrestricted use, distribution, and reproduction in any medium, provided you give appropriate credit to the original author(s) and the source, provide a link to the Creative Commons license, and indicate if changes were made.

\section{References}

Bassolino M, Serino A, Ubaldi S, Làdavas E (2010) Everyday use of the computer mouse extends peripersonal space representation. Neuropsychologia 48:803-811. https://doi.org/10.1016/j. neuropsychologia.2009.11.009

Bassolino M, Finisguerra A, Canzoneri E et al (2014) Dissociating effect of upper limb non-use and overuse on space and body representations. Neuropsychologia 70:385-392. https://doi. org/10.1016/j.neuropsychologia.2014.11.028

Bremmer F, Schlack A, Shah NJ et al (2001) Polymodal motion processing in posterior parietal and premotor cortex: a human fMRI study strongly implies equivalencies between humans and monkeys. Neuron 29:287-296

Brozzoli C, Cardinali L, Pavani F et al (2010) Neuropsychologia action-specific remapping of peripersonal space. Neuropsychologia 48:796-802. https://doi.org/10.1016/j. neuropsychologia.2009.10.009

Brozzoli C, Gentile G, Ehrsson HH (2012a) That's near my hand! Parietal and premotor coding of hand-centered space contributes to localization and self-attribution of the hand. J Neurosci 32:1457314582. https://doi.org/10.1523/JNEUROSCI.2660-12.2012

Brozzoli C, Makin TR, Cardinali L et al (2012b) Peripersonal space: a multisensory interface for body-object interactions. In: Murray MM, Wallace MT (eds) The neural bases of multisensory processes. CRC Press, Boca Raton, pp 449-466

Brozzoli C, Gentile G, Bergouignan L, Ehrsson HH (2013) A shared representation of the space near oneself and others in the human premotor cortex. Curr Biol 23:1764-1768. https://doi. org/10.1016/j.cub.2013.07.004

Canzoneri E, Magosso E, Serino A (2012) Dynamic sounds capture the boundaries of peripersonal space representation in humans. PLoS One 7:e44306. https://doi.org/10.1371/journal.pone.0044306

Canzoneri E, Marzolla M, Amoresano A et al (2013a) Amputation and prosthesis implantation shape body and peripersonal space representations. Sci Rep 3:2844
Canzoneri E, Ubaldi S, Rastelli V et al (2013b) Tool-use reshapes the boundaries of body and peripersonal space representations. Exp Brain Res 228:25-42. https://doi.org/10.1007/s00221-013-3532-2

Cardinali L, Brozzoli C, Farnè A (2009) Peripersonal space and body schema: two labels for the same concept? Brain Topogr 21:252 260. https://doi.org/10.1007/s10548-009-0092-7

Cléry J, Guipponi O, Wardak C, Ben Hamed S (2015) Neuronal bases of peripersonal and extrapersonal spaces, their plasticity and their dynamics: knowns and unknowns. Neuropsychologia 70:313-326. https://doi.org/10.1016/j. neuropsychologia.2014.10.022

Colby CL, Duhamel J-R, Goldberg ME (1993) Ventral intraparietal area of the macaque: anatomic location and visual response properties. J Neurophysiol 69:902-914

Cooke DF, Graziano MS (2003) Defensive movements evoked by air puff in monkeys. J Neurophysiol 90:3317-3329

Cooke DF, Graziano MSA (2004) Sensorimotor integration in the precentral gyrus: polysensory neurons and defensive movements. J Neurophysiol 91:1648-1660

de Haan AM, De Smit M, Van Der Stigchel S (2016) Approaching threat modulates visuotactile interactions in peripersonal space. Exp Brain Res 234:1875-1884. https://doi.org/10.1007/ s00221-016-4571-2

de Vignemont F, Iannetti GD (2015) How many peripersonal spaces? Neuropsychologia 70:327-334. https://doi.org/10.1016/j. neuropsychologia.2014.11.018

Duhamel J-R, Colby CL, Goldberg ME (1998) Ventral intraparietal area of the macaque: congruent visual and somatic response properties. J Neurophysiol 79:126-136

Ferri F, Costantini M, Huang Z et al (2015a) Intertrial variability in the premotor cortex accounts for individual differences in peripersonal space. J Neurosci 35:16328-16339. https://doi.org/10.1523/ JNEUROSCI.1696-15.2015

Ferri F, Tajadura-Jiménez A, Väljamäe A et al (2015b) Emotioninducing approaching sounds shape the boundaries of multisensory peripersonal space. Neuropsychologia 70:468-475. https:// doi.org/10.1016/j.neuropsychologia.2015.03.001

Fogassi L, Gallese V, Fadiga L et al (1996) Coding of peripersonal space in inferior premotor cortex (area F4). J Neurophysiol 76:141-157

Galli G, Noel JP, Canzoneri E et al (2015) The wheelchair as a fullbody tool extending the peripersonal space. Front Psychol 6:1-11. https://doi.org/10.3389/fpsyg.2015.00639

Gentile G, Petkova VI, Ehrsson HH (2011) Integration of visual and tactile signals from the hand in the human brain: an fMRI study. J Neurophysiol 105:910-922. https://doi.org/10.1152/ jn.00840.2010

Graziano MS (1999) Where is my arm? The relative role of vision and proprioception in the neuronal representation of limb position. Proc Natl Acad Sci USA 96:10418-10421. https://doi. org/10.1073/pnas.96.18.10418

Graziano MSA, Cooke DF (2006) Parieto-frontal interactions, personal space, and defensive behavior. Neuropsychologia 44:2621-2635. https://doi.org/10.1016/j.neuropsychologia.2005.09.011

Graziano MS, Gross CG (1995) The representation of extrapersonal space: a possible role for bimodal, visual-tactile neurons. In: Gazzaniga MS (ed) The cognitive neurosciences. MIT Press, Cambridge (MA), pp 1021-1034

Graziano M, Yap GS, Gross CG (1994) Coding of visual space by premotor neurons. Science (80-) 226:1054-1057

Graziano MS, Hu XT, Gross CG (1997) Visuospatial properties of ventral premotor cortex. J Neurophysiol 77:2268-2292

Graziano MSA, Reiss LAJ, Gross CG (1999) A neuronal representation of the location of nearby sounds. Nature 397:428-430

Grivaz P, Blanke O, Serino A (2017) Common and distinct brain regions processing multisensory bodily signals for peripersonal 
space and body ownership. Neuroimage 147:602-618. https://doi. org/10.1016/j.neuroimage.2016.12.052

Haegens S, Handel BF, Jensen O (2011) Top-down controlled alpha band activity in somatosensory areas determines behavioral performance in a discrimination task. J Neurosci 31:5197-5204. https://doi.org/10.1523/JNEUROSCI.5199-10.2011

Hunley SB, Marker AM, Lourenco SF (2017) Individual differences in the flexibility of peripersonal space. Exp Psychol 64:49-55. https://doi.org/10.1027/1618-3169/a000350

Hyvärinen J (1981) Regional distribution of functions in parietal association area 7 of the monkey. Brain Res 206:287-303

Jiang H-H, Hu Y-Z, Wang J-H et al (2013) Visuospatial properties of caudal area 7b in Macaca fascicularis. Zool Res 34:E50-E61. https://doi.org/10.3724/SP.J.1141.2013.E02E50

Kandula M, Hofman D, Dijkerman HC (2015) Visuo-tactile interactions are dependent on the predictive value of the visual stimulus. Neuropsychologia 70:358-366. https://doi.org/10.1016/j. neuropsychologia.2014.12.008

Kandula M, van der Stoep N, Hofman D, Dijkerman HC (2017) On the contribution of overt tactile expectations to visuo-tactile interactions within the peripersonal space. Exp Brain Res 235:2511-2522

Kurata K (1989) Distribution of neurons with set- and movementrelated activity before hand and foot movements in the premotor cortex of rhesus monkeys. Exp Brain Res 77:245-256. https://doi. org/10.1007/BF00274982

Kurata K, Okano K, Tanji J (1985) Distribution of neurons related to a hindlimb as opposed to forelimb movement in the monkey premotor cortex. Exp Brain Res 60:188-191

Làdavas E, di Pellegrino G, Farnè A, Zeloni G (1998) Neuropsychological evidence of an integrated visuotactile representation of peripersonal space in humans. J Cogn Neurosci 10:581-589. https://doi.org/10.1162/089892998562988

Làdavas E, Pavani F, Farnè A (2001) Auditory peripersonal space in humans: a case of auditory-tactile extinction. Neurocase 7:97103. https://doi.org/10.1093/neucas/7.2.97

Makin TR, Holmes NP, Zohary E (2007) Is that near my hand? Multisensory representation of peripersonal space in human intraparietal sulcus. J Neurosci 27:731-740. https://doi.org/10.1523/ JNEUROSCI.3653-06.2007

Makin TR, Holmes NP, Ehrsson HH (2008) On the other hand: dummy hands and peripersonal space. Behav Brain Res 191:1-10. https:// doi.org/10.1016/j.bbr.2008.02.041

Muakkassa KF, Strick PL (1979) Frontal lobe inputs to primate motor cortex: evidence for four somatotopically organized "premotor" areas. Brain Res 177:176-182. https://doi. org/10.1016/0006-8993(79)90928-4

Noel J, Wallace M (2016) Relative contributions of visual and auditory spatial representations to tactile localization. Neuropsychologia 82:84-90. https://doi.org/10.1016/j. neuropsychologia.2016.01.005

Noel JP, Grivaz P, Marmaroli P et al (2015) Full body action remapping of peripersonal space: the case of walking. Neuropsychologia 70:375-384. https://doi.org/10.1016/j. neuropsychologia.2014.08.030

Noel J, Marta Ł, Wallace M, Serino A (2016) Multisensory simultaneity judgment and proximity to the body. J Vis $16: 1-17$. https://doi. org/10.1167/16.3.21

Pozeg P, Galli G, Blanke O (2015) Those are your legs: the effect of visuo-spatial viewpoint on visuo-tactile integration and body ownership. Front Psychol 6:1-14. https://doi.org/10.3389/ fpsyg.2015.01749

Rizzolatti G, Scandolara C, Matelli M, Gentilucci M (1981a) Afferent properties of periarcuate neurons in macaque monkeys II. Visual responses. Behav Brain Res 2:147-163
Rizzolatti G, Scandolara C, Matelli M, Gentilucci M (1981b) Afferent properties of periarcuate neurons in macaque monkeys. I. Somatosensory responses. Behav Brain Res 2:125-146. https://doi. org/10.1016/0166-4328(81)90052-8

Rizzolatti G, Fadiga L, Fogassi L, Gallese V (1997) The space around us. Science (80-) 227:190-191

Salomon R, Noel JP, Łukowska M et al (2017) Unconscious integration of multisensory bodily inputs in the peripersonal space shapes bodily self-consciousness. Cognition 166:174-183. https://doi. org/10.1016/j.cognition.2017.05.028

Sambo CF, Iannetti GD (2013) Better safe than sorry? The safety margin surrounding the body is increased by anxiety. J Neurosci 33:14225-14230. https://doi.org/10.1523/ JNEUROSCI.0706-13.2013

Scandola M, Aglioti SM, Bonente C et al (2016) Spinal cord lesions shrink peripersonal space around the feet, passive mobilization of paraplegic limbs restores it. Sci Rep 6:24126. https://doi. org/10.1038/srep24126

Schicke T, Röder B (2006) Spatial remapping of touch: confusion of perceived stimulus order across hand and foot. Proc Natl Acad Sci USA 103:11808-11813. https://doi.org/10.1073/pnas.0601486103

Schicke T, Bauer F, Röder B (2009) Interactions of different body parts in peripersonal space: how vision of the foot influences tactile perception at the hand. Exp Brain Res 192:703-715. https://doi. org/10.1007/s00221-008-1587-2

Sekiya N, Nagasaki H, Ito H, Furuna T (1997) Optimal walking in terms of variability in step length. J Orthop Sports Phys Ther $26: 266-272$

Serino A, Annella L, Avenanti A (2009) Motor properties of peripersonal space in humans. PLoS One 4:1-8. https://doi.org/10.1371/ journal.pone.0006582

Serino A, Canzoneri E, Avenanti A (2011) Fronto-parietal areas necessary for a multisensory representation of peripersonal space in humans: an rTMS study. J Cogn Neurosci 23:2956-2967

Serino S, Noel J-P, Galli G et al (2015) Body part-centered and full body-centered peripersonal space representation. Sci Rep. https:// doi.org/10.1038/srep18603

Shorter KA, Wu A, Kuo AD (2017) The high cost of swing leg circumduction during human walking. Gait Posture 54:265-270. https:// doi.org/10.1016/j.gaitpost.2017.03.021

Stettler BA, Thomas LE (2016) Visual processing is biased in peripersonal foot space. Atten Percept Psychophys. https://doi. org/10.3758/s13414-016-1225-1

Taffou M, Viaud-Delmon I (2014) Cynophobic fear adaptively extends peri-personal space. Front Psychiatry 5:3-9. https://doi. org/10.3389/fpsyt.2014.00122

Teneggi C, Canzoneri E, Pellegrino G et al (2013) Social modulation of peripersonal space boundaries. Curr Biol 23:406-411. https:// doi.org/10.1016/j.cub.2013.01.043

Terrier P, Schutz Y (2003) Variability of gait patterns during unconstrained walking assessed by satellite positioning (GPS). Eur J Appl Physiol 90:554-561. https://doi.org/10.1007/ s00421-003-0906-3

Terrier P, Turner V, Schutz Y (2005) GPS analysis of human locomotion: further evidence for long-range correlations in stride-tostride fluctuations of gait parameters. Hum Mov Sci 24:97-115. https://doi.org/10.1016/j.humov.2005.03.002

Van Elk M, Forget J, Blanke O (2013) The effect of limb crossing and limb congruency on multisensory integration in peripersonal space for the upper and lower extremities. Conscious Cogn 22:545-555. https://doi.org/10.1016/j.concog.2013.02.006 